\title{
EFFECT OF APDT ON HUMAN DENTAL BIOFILM ISOLATED STREPTOCOCCI SPECIES WITH 2 PHOTOSENSITIZERS - IN VITRO STUDY
}

\author{
Maya Doychinova ${ }^{1}$, Vesselin Kussovski² ${ }^{2}$, Slavcho Dimitrov ${ }^{1}$ \\ ${ }^{1}$ Department of Conservative Dental Treatment and Oral Pathology, \\ Faculty of Dental Medicine, Medical University of Varna \\ ${ }^{2}$ Bulgarian Academy of Sciences
}

\begin{abstract}
AIM: The aim of this study is to evaluate and compare the in vitro antimicrobial effect of 2 photosensitizers: FotoSan medium and ZnPcMe on a biofilm isolate of clinical strain of Streptococci species .

MATERIALS AND METHODS: Twelve patients were instructed to stop using any kind of oral hygiene care for $\mathbf{2 4}$ hours. Plaque was collected with sterile dental floss, placed in vial containing $3 \mathrm{ml}$ of BFS. Tenfold dilution of the suspension was made. Samples of $0.1 \mathrm{ml}$ were plated on Mitis Salivarius agar. Bacteria were Gram-stained as well and underwent morphological identification. A bacterial suspension at a density of $10^{6}$ cells $/ \mathrm{ml}$ was used. An incubation mixture of $1 \mathrm{ml}$ bacterial suspension $\left(10^{6} \mathrm{CFU} / \mathrm{ml}\right)$ and the corresponding photosensitizer were prepared, achieving a different final concentration of PS. LED light source with a wavelength of $643 \mathrm{~nm}$ was used for irradiation. Samples and controls for each tested PS after $24 \mathrm{~h}$ incubation at $37^{\circ} \mathrm{C}$ were reported.

RESULTS: The control samples in dark showed a minor antimicrobial effect. Samples of bacterial suspension + PS + light irradiation expressed statistically relevant antibacterial effect on Streptococci species with small differences in the different concentrations and irradiation time used.

CONCLUSION: The clinical strain of Streptococci species was susceptible to photodynamic inactivation by the used photosensitizers: FotoSan ${ }^{\oplus}$ edium and ZnPcMe. Full photoinactivation was not observed. Dark toxicity was to be neglected. ZnPcMe should undergo further trials and given the positive photodynamic effect in vitro, be licensed for clinical use. FotoSan ${ }^{\oplus}$ medium should undergo further in vivo and in vitro experiments in order to establish an alternative protocol for caries prevention.
\end{abstract}

Keywords: dental biofilm, photosensitizers

Address for correspondence:

Maya Doychinova

Faculty of Dental Medicine

Medical University of Varna

84 Tsar Osvoboditel Blvd

9000 Varna

e-mail:mayunid@yahoo.com

Received: April 19, 2017

Accepted: June 29, 2017

\section{INTRODUCTION}

Dental caries is a disease with a pandemic spread and for the last 20 years has been a part of the entities with social impact. Therefore, the issues concerning its prevention and etiology control are always relevant. Dental caries prevention aims at balancing the multiple factors considered causative agents for the "localized, post-eruptive, pathological process of external origin involving softening of 
Effect of APDT on Human Dental Biofilm Isolated Streptococci Species with 2 Photosensitizers - In Vitro Study

the hard tooth tissue and proceeding to the formation of a cavity." This quote is the definition given by the World Health Organization. The conditions for the onset and development of a carious lesion are microorganisms, carbohydrate, primarily sucrose, and a susceptible tooth surface.

Among all the oral bacteria, Mutans Streptococ$c i$, colonize the dental surface, contribute largely to the formation of the biofilm matrix and are considered causative agents of dental caries in humans in the presence of fermentable carbohydrates e.g., sucrose and fructose (1). Mutans Streptococci belong to the group of oral Streptococci species. On the other hand, researches show the connections and the ecologic relationships in the complex structure of the oral dental biofilm. It appears that if the dental surfaces are primary colonized by $S$. mutans its final count in the dental biofilm will exceed greatly the number of S. sobrinus and other Streptococci, such as S. Gordoni, S. pyogenes, S. oralis, S. Mitis, S. pneumoniae, S. cristatus, S. parasanguinis, S. sanguinis, S. Sobrinus known as S. species. The opposite is also true e.g. if S.sobrinus are chronologically the first species among the oral streptococcal population, then the total count of S.mutans will be significantly lower compared to other Streptococci (2).

We could summarize that dental caries is biofilm-mediated disease, but not a classic infection. Infections caused by biofilm-forming bacteria are often difficult to treat. Biofilm formation almost always leads to a large increase in resistance to antimicrobial agents (up to 1000 -fold decrease in susceptibility) in comparison with planktonic cultures grown in conventional liquid media (3) .

Photodynamic therapy is a particularly suitable method for use in dentistry because of the anatomical features of the region, providing a relatively easy access to light exposure and for topical administration of the photoactive substance with an impact on organized in biofilm bacteria $(4,5)$. Worldwide, the antimicrobial photodynamic therapy (APDT) in dentistry is successful and shows potential in the treatment of bacterial, fungal and viral infections.

The possibilities of APDT for the control of cariogenic biofilm has recently been explored. Efforts are focused in several directions: $\diamond$ Study of the sensitivity of Streptococcus mutans - laboratory strains, most commonly in the form of planktonic cells and on in vitro biofilm models established.

$\diamond$ Experimental study on the effect of different photosensitiserson clinical isolates of MS and Streptococci species.

$\diamond$ There have been studies to compare the photodynamic effect under the same parameters in APDT but respectively on one-, two- and threespecies biofilm created in vitro.

\section{MATERIALS AND METHODS}

We used: sterile dental floss, a vial containing BFS, Mitis salivarius agar, sucrose, FotoSan-TBO $180 \mu \mathrm{M}, 90 \mu \mathrm{M}$ and $30 \mu \mathrm{M}, \mathrm{ZnPcMe}$ - Phthalocyanine zinc (II) complex: 2,9,16,23-tetrakis(3-methylpyridyloxy)phthalocyanine zinc(II) - ZnPcMe $-15 \mu \mathrm{M}$, $40 \mu \mathrm{M}$, LED-643nm wavelength, ELO Ltd - Bulgaria. The irradiation characteristics were as follows: power density $100 \mathrm{~mW} / \mathrm{cm}^{2}$ (controlled with photometer Spectra Physics, USA), light dose $12 \mathrm{~J} / \mathrm{cm}^{2}$ (exposure time - $2 \mathrm{~min}$ ) and $60 \mathrm{~J} / \mathrm{cm}$ (exposure time - $10 \mathrm{~min}$ ), and a wide-field dissecting microscope.

Selective medium for Streptococci species was Mitis-salivarius agar, sucrose. The medium was heated to dissolve the components and then autoclaved at $121^{\circ} \mathrm{C}$ for 15 minutes. The medium was then cooled at $45^{\circ} \mathrm{C}$.

Dental plaque was collected from 12 people with sterile dental floss. Samples were taken from the interdental spaces. All participants followed the instructions to discontinue interdental oral hygiene for 24 hours in order for sufficient amount of dental plaque to accumulate interdentally. Forms of informed consent were signed. Professional oral hygiene and instruction for personal oral hygiene were given to each of the participants after collecting the samples. They were scheduled for control in 7 days.

The dental floss samples were immediately placed in a small screw-capped vial containing $3 \mathrm{ml}$ BMS as transport medium. The vials were agitated on a vortex test tube mixer for $30 \mathrm{sec}$ in order to dislodge the plaque from the floss and to obtain homogeneous suspension. Tenfold dilutions of the suspensions were made in $0.05 \%$ yeast extract water. Duplicate $0.1 \mathrm{ml}$ samples of the dilutions were plated 
Maya Doychinova, Vesselin Kussovski, Slavcho Dimitrov1

in a selective medium for Streptococci species as described above and spread on the medium. All plates were incubated under $\mathrm{CO}_{2}$ conditions - "candle jar" technique, for 24 hours at $37^{\circ} \mathrm{C}$. After incubation, the plates were allowed to stand at room temperature for 2 hours. The plates were then examined. Four of the samples were predominated by other bacterial growth like colonies of the enterococci-dark blue to brown and flat, as well as yeast, appearing as large white to light blue matte colonies. The other eight had sufficient colonies of Streptococci species that underwent further morphological identification.

A bacterial suspension at a density of $10^{6}$ cells/ $\mathrm{ml}$ is used for the needs of antimicrobial photodynamic therapy. Samples of microbial suspensions of each participant were incubated for $20 \mathrm{~min}$ in the dark with:

$\diamond 30 \mu \mathrm{M}, 90 \mu \mathrm{M}, 180 \mu \mathrm{M}$ FotoSan ${ }^{\oplus}$

$\diamond 15 \mu \mathrm{M}, 40 \mu \mathrm{M} \mathrm{ZnMePc}$

Controls we as follows:

$\diamond$ bacterial suspension in the dark;

$\diamond$ bacterial suspension + PS in the dark - dark toxicity sample;

$\diamond$ bacterial suspension + PS + light - true photodynamic sample.

\section{Sample:}

Bacterial suspension (BS) + PS + irradiation. $200 \mu \mathrm{l}$ of the mixture (bacterial suspension + PS), after incubation in the dark for $20 \mathrm{~min}$, were placed in a standard 96-well polystyrene microtiter plate, where the irradiation was performed. LED source with a wavelength of $643 \mathrm{~nm}$ was applied with irradiance of $100 \mathrm{~mW} / \mathrm{cm}^{-2}$ controlled during the experiment by photometer (Spectra Physics, USA) for the needed for the group irradiation time.

After the irradiation, $0.1 \mathrm{~mL}$ samples were taken off and serially diluted (10-fold) with phosphate- buffered saline (PBS), $\mathrm{pH} 7.4,\left(10^{6} ; 10^{5} ; 10^{4} ; 10^{3} ; 10^{2}\right.$;). Aliquots $(0.025 \mathrm{ml})$ were spread over the prepared selective medium. The number of colonies (CFU) formed on each plate was counted following $48 \mathrm{~h}$ incubation at $37^{\circ} \mathrm{C}$ in $5 \% \mathrm{CO}_{2}$.

\section{Statistics:}

Each experiment was carried out in duplicate and the data are presented as a mean \pm standard deviation (SD). The difference between two means was compared by a two-tailed unpaired Student's test. The values of $\mathrm{P}<0.05$ were considered as significant.

\section{RESULTS}

Examination of the number of the colonies (CFU) that have grown in Petri dishes serves to analyze the effect of the two PSs on Streptococci species group, subject of our study. The photodynamic inactivation of $10^{6} \mathrm{CFU} \cdot \mathrm{ml}^{-1}$ bacterial suspensions of Streptococci species clinical strain was compared for 2 different PSs-2, 9, 16, 23-tetrakis (3-methylpyridyloxy) phthalocyanine zinc (II) - ZnPcMe and FotoSan-medium. Given the results of our previous study about the photodynamic effect of FotoSan ${ }^{\odot}$ the used concentrations in this study were $30 \mu \mathrm{M}$, $90 \mu \mathrm{M}, 180 \mu \mathrm{M}$. The concentration used for $\mathrm{ZnPcMe}$ were $40 \mu \mathrm{M}$ and $15 \mu \mathrm{M}$. We consider as pilot experiments, the tests done with FotoSan ${ }^{\oplus}$ medium $180 \mu \mathrm{M}$ and $\mathrm{ZnPcMe} 40 \mu \mathrm{M}$. In both, regardless of the time of irradiation, 2, 10, 15 minutes, we observed full photo-inactivation. The samples of bacterial suspension + PS + LED irradiation showed statistically relevant antibacterial effect on Streptococci species, clinical strain with small differences in the tested PSs. Dark toxicity was pronounced when $180 \mu \mathrm{M}$ Fotosan and $40 \mu \mathrm{M}$ of $\mathrm{ZnPcMe}$ was used - a 4 log decrease of CFU.ml ${ }^{-1}$. The control samples made in the dark for the main experiments show negligible dark toxicity at all dye concentrations used $(<0.5 \log$ decrease

Table. 1. Effect of fotosan on S.species

\begin{tabular}{lcc|c} 
Light & \multicolumn{3}{c}{ Streptococci species $\left(\mathrm{CFU} \cdot \mathrm{ml}^{-1} / \pm \mathrm{SD} /\right)$} \\
& $(-)$ Fotosan & $\mathbf{3 0 \mu M}$ Fotosan & $\mathbf{9 0 \mu M}$ Fotosan \\
$1 .(-)$ & $(2.94 \pm 0.62) \times 10^{6}$ & $(2.45 \pm 0.41) \times 10^{6}$ & $(1.76 \pm 0.35) \times 10^{6}$ \\
$2.2 \mathrm{~min}$ & $(2.43 \pm 0.97) \times 10^{6}$ & $(6.60 \pm 1.61) \times 10^{4}$ & $(5.10 \pm 1.40) \times 10^{3}$ \\
& $p 2-1=0.48$ & $p 2-1=0.0005$ & $p 2-1=0.0009$ \\
$3.5 \mathrm{~min}$ & $(2.24 \pm 0.41) \times 10^{6}$ & $(8.10 \pm 2.60) \times 10^{3}$ & $(3.10 \pm 1.90) \times 10^{3}$ \\
& $p 3-1=0.17$ & $p 3-1=0.0004$ & $p 3-1=0.0009$
\end{tabular}

Scripta Scientifica Medicinae Dentalis, vol. 3, No 1, 2017, pp. 47-51 
Effect of APDT on Human Dental Biofilm Isolated Streptococci Species with 2 Photosensitizers - In Vitro Study

Table 2. Effect of Zn-phtalocyanin on S. species

\begin{tabular}{lc} 
& CFU/ml \\
\cline { 2 - 2 } Sample & Streptococcus species \\
1.(-) c Zn-phtalocyanine, & $(1.45 \pm 0.66) \times 10^{6}$ \\
(-) light & $(7.72 \pm 2.39) \times 10^{5}$ \\
2. (+) c Zn-phtalocyanine, & $p 2-1=0.1$ \\
(-) light & $p 2-3=0.002$ \\
& $(6.30 \pm 2.50) \times 10^{1}$ \\
3.(+) c Zn-phtalocyanine, & $p 3-1=0.01$ \\
2 min light & $p 3-2=0.002$ \\
& 0 \\
4.(+) c Zn-phtalocyanine, & $p 4-1=0.01$ \\
5 min light & $p 4-2=0.002$ \\
\hline \hline
\end{tabular}

of viable cells), except for $90 \mu \mathrm{M}$ Fotosan ${ }^{\oplus}(1 \log$ decrease of viable cells).

\section{DISCUSSION}

Different experimental approaches seek to determine the most effective, non-toxic, economical and short approach to controlling Streptococcus mutans and the created by it and Streptococci species, biofilms. We have tested various concentrations and incubation time during the irradiation with the light source.

Pereira et al. report the treatment of a clinically isolated strain of Streptococcus mutans with Erythrosine (ERI) $2 \mu \mathrm{M}$ and Rose Begawan (RB) $2 \mu \mathrm{M}$ and LED (440-460nm) exposure. The authors reported a $5.16 \log$ reduction for ERI and a $6.86 \log$ reduction for $\mathrm{RB}$ CFU/ml (6).

We think that alternative ways of controlling the number of $S$. mutans in dental biofilms is needed in order to promote better dental health, less carious lesions and less endodontic complications due to advanced dental caries. This approach could become an alternative to the well spread fluoride prophylaxis of dental caries. Among these alternative antimicrobial methods, APDT represents a modern method of choice.

In this study we aim to analyze the effect on two different PSs activated with LED-generated light on clinically isolated from human dental biofilm, Streptococci species.

The potential of APDT is just beginning to be explored and fully understood. Its possible applica- tions in the management of the oral cavity conditions and diseases, as an alternative or an additional modality for the conventional means and protocols have been confirmed in the recent years by numerous studies (7).

The areas of interest include: endodontic space disinfection, treatment of primary and secondary dental caries, and treatment of periodontal acute and chronic conditions, peri-implantitis. We consider as unexplored its plausible and beneficial use is in the field of caries prevention and the management of early, non-cavitated, caries lesions.

Embracing the knowledge, that biofilm derived bacteria differ by certain behavioral characteristic, e.g. susceptibility to antimicrobial drugs, from the planktonic and reference strains, we aimed at establishing the effect of APDT on clinic strain Streptococci species. Our target was chosen based on the etiology of dental caries as a multifactorial disease where the presence of acidogenic bacteria is the crucial point leading to demineralization. The disproportional increase of Mutans Streptococci creates not only the $\mathrm{pH}$ needed for initial demineralization, but also contributes in two other directions. On the one hand the drop of $\mathrm{pH}$ initially makes a perfect condition for the flourishing of Mutans Streptococci population, and on the other it gives the chance of other bacteria, non-Mutans Streptococci, Veilonella, and others to gain cariogenic potential thus maintaining and complicating the demineralization process.

The clinical strain Streptococci species showed excellent susceptibility to the 2 PSs used by us in the study. The results are comparable and we consider as contribution the following facts:

1. Even though clinically tested and approved, the FotoSan ${ }^{\otimes}$ with active substance toluidine blue was mostly tested and used with laser light source. In his study we confirmed its excellent photo inactivating ability by inducing photodynamic effect with LED light source. This will make the protocol for caries prevention and management or incipient caries less expensive and more affordable.

2. The synthesized by us ZnPcMe has no dark toxicity in the used concentrations on the Streptococci species clinical strain. The susceptibility of Streptococci species, on the other hand, when 
Maya Doychinova, Vesselin Kussovski, Slavcho Dimitrov1

LED light is applied for 5, as well as for 2 minutes, is a good base for further clinical tests and trials in order to assess its non-risky in vivo application for caries prevention and early caries management.

In our opinion, APDT could be incorporated as an alternative or an adjuvant modality in all areas where the side effects of conventional antibiotic therapy and the bacterial resistance to antibiotics are likely to occur.

\section{CONCLUSION}

The Streptococci species clinical strain is susceptible to APDT with FotoSan medium and the synthesized by us $\mathrm{ZnPcMe}$. Due to the pronounced dark toxicity at the concentration of $180 \mu \mathrm{M}$ we used less concentrated suspension of $30 \mu \mathrm{M}$ and $90 \mu \mathrm{M}$ and both showed excellent photodynamic inactivation, regardless of the period $f$ LED irradiation- 2 or 5 minutes.

As far as $\mathrm{ZnPcMe}$ is concerned, the preliminary tests with $40 \mu \mathrm{M}$ showed full photoinactivation. Our goal was to minimize the concentration and to achieve an active concentration for the clinically accepted period of irradiation 2 and 5 minutes. The results show that at $15 \mu \mathrm{M}$ concentration there is full photoinactivation for both time intervals.

Therefore, we consider Fotosan medium (active substance toluidine blue) $30 \mu \mathrm{M}$ and $90 \mu \mathrm{M}$ as suitable for in vivo tests, concerning the early plaque formation. The goal of such a study would be to establish an alternative protocol for caries prevention and management of incipient caries. We consider $\mathrm{ZnPcMe}$ as potential photosensitizer for alternative management of incipient caries as well as for caries prevention. In our opinion, the $\mathrm{ZnPcMe}$ should undergo further trials and given the positive photodynamic effect in vitro, be licensed for clinical use.

\section{REFERENCES}

1. Kleinberg I. A mixed-bacteria ecological approach to understanding the role of the oral bacteria in dental caries causation: an alternative to Streptococcus mutans and the specific-plaque hypothesis. Crit Rev Oral Biol Med. 2002; 13(2):108-25. doi: $10.1177 / 154411130201300202$

2. Schaeken MJ, Creugers T, Van der Hoeven

JS. Relationship between dental plaque indi- ces and bacteria in dental plaque and those in saliva. J Dent Res. 1987; 66(9):1499-502. doi: $10.1177 / 00220345870660091701$

3. Taraszkiewicz A, Fila G, Grinholc M, Nakonieizna J. Innovative strategies to overcome biofilm resistance. 2013. BioMed Research Int; 2013. doi: $10.1155 / 2013 / 150653$

4. Wilson M, Burns T, Pratten J. Killing of Streptococcus sanguis in biofilms using a light-activated antimicrobial agent. J Antimicrob Chemother. 1996; 37(2):377-81. doi: 10.1093/jac/37.2.377

5. Zanin IC, Gonçalves RB, Junior AB, Hope CK, Pratten J. Susceptibility of Streptococcus mutans biofilms to photodynamic therapy: an in vitro study. J Antimicrob Chemother. 2005 Aug; 56(2):324-30. doi: 10.1093/jac/dki232

6. Pereira CA, Romerio R, Costa A, Machado A, Junqueira J, Jorge A. Susceptibility of Candida albicans, Staphylococcus aureus, and Streptococcus mutans biofilms to photodynamic inactivation: an in vitro study. Lasers Med Sci. 2011 May; 26(3):3418. doi:10.1007/s10103-010-0852-3

7. Wood S, Nattress B, Kirkham J, Shore R, Brookes $\mathrm{S}$, Griffiths J, et al.An in vitro study of the use of photodynamic therapy for the treatment of natural oral plaque biofilms formed in vivo. J Photochem Photobiol B. 1999;50(1):1-7. doi: 10.1016/ S1011-1344(99)00056-1

Acknowledgements:

To the National Science Fund, Sofia, Bulgaria for the Grants DO-02-177/88 